\title{
Existence and Approximation Results to the Cauchy Problem for a Class of Differential-Algebraic Equations
}

\author{
K. R. SCHNEIDER
}

For a class of differential-algebraic equations arising for example in modelling electrical circuits conditions are derived which ensure the existence of a unique solution of the Cauchy problem on any finite interval and its computation by means of a wave-form relaxation algorithm in case of a large system. The solution concept is understood in the sense of Caratheodory.

Key words : differential-algebraic equations, Carathéodory solution, wave-form relaxarion AMS subject classification: $34 \wedge 34,58 \mathrm{~F} 99,65 \mathrm{~L}$ 0S

\section{Introduction}

This paper is concerned with the initial value problem for differential-algebraic systems of the form

$$
\frac{d x}{d t}=F\left(x, z, \frac{d x}{d t}, t\right), z=G\left(x, z, \frac{d x}{d t}, t\right), x\left(t_{0}\right)=x_{0}, t \in J:=\left[t_{0}, t_{0}+T\right],
$$

where $x$ is an $n$-vector, $z$ is an $m$-vector, and $T$ is a given positive number. We are interested in conditions guaranteeing the existence of a unique solution $(\bar{x}, \bar{z})$ of $(1.1)$ and in iterative procedures approaching $(\bar{x}, \bar{z})$ in case $n+m$ to be large. The problem formulated above arises for example in modelling non-linear electrical networks [6]. By using Kirchhoff's laws, the underlying constitutive relations (voltage-current relations) and the corresponding dynamic equations we arrive at a system of the kind

$$
H(d y / d t, y, t)=0 \text {, }
$$

where the vector $y$ of network variables consists of two components $x$ and $z$ and the derivative of $z$ does not occur in (1.2). Thus, without loss of generality we may represent the system (1.2) in the form (1.1).

By applying traditional integration procedures to solve the initial value problem (1.1) the computing time growths rapidly when $n+m$ becomes large. From this reason, new methods for numerical treatment of such problems has been developed basing on the decomposition either of the corresponding large system of linear equations in the process of discretization or of the corresponding differential-algebraic system (1.1) itself. In the latter case this method is called wave-form relaxation method [1,3-5]. For a broad class of wave-form relaxation methods the canonical iteration scheme reads

$$
\begin{aligned}
& d x^{k} / d t=\widetilde{F}\left(x^{k}, x^{k-1}, z^{k-1}, d x^{k-1 / d t, t}\right) \\
& z^{k}=\widetilde{G}\left(x^{k}, x^{k}, z^{k-1}, d x^{k-1 / d t, t}\right) \quad x^{k}\left(t_{0}\right)=x_{0}, t \in J .
\end{aligned}
$$

The convergence of this scheme in some Banach space was proved in [3] under a crucial assumption whcse verification is not obvious. In this paper we derive an explicite con dition on the Lipschitz constants of $F$ and $G$ which implies the convergence of (1.3). At the same time we give a new short proof of the convergence of (1.3) and introduce a solution concept. the so-called Caratheodory solution which is more appropriate for applications. 


\section{Notation and definitions}

Let $|\cdot|$ be the Euclidean norm, $L^{P}\left(J, \mathbb{R}^{k}\right)$ the space of functions $z: J \rightarrow \mathbb{R}^{k}$ such that $|z|^{k}$ is integrable in the sense of Lebesgue, and $L^{\infty}\left(J, R^{k}\right)$ the set of functions $z: J \rightarrow R^{k}$ the essential supremum (ess sup) of which is bounded. Further, let $C\left(J, R^{k}\right)$ be the space of continuous functions $z: J \rightarrow \mathbf{R}^{k}, A C\left(J, \mathbf{R}^{k}\right)$ the set of all functions $z \in C\left(J, \mathbf{R}^{k}\right)$ which are absolutely continuous

Definition 2.1: Let $D$ be a set in $\mathbf{R}^{k}$. A function $f: D \times J \rightarrow \mathbf{R}^{l}$ is said to satisfy the Caratheodory condition if

(i) $f$ is defined for all $x \in D$ and for almost all (f.a.a.) $t \in J$,

(ii) $f$ is continuous in $x$ f.a.a. $t \in J$ and (Lebesgue) measurable in $t$ for all $x \in D$.

Definition 2.2: The couple $(\bar{x}, \bar{z})$ is said to be a solution of $(1.1)$ in the sense of Caratheodory ( $\mathrm{Ca}$-solution) if

(i) $(\bar{x}, \bar{z}) \in A C\left(J, \mathbf{R}^{n}\right) \times L^{1}\left(J, \mathbf{R}^{m}\right)$,

(ii) $(\bar{x}, \bar{z})$ satisfies (1.1) f.a.a. $t \in J$,

(iii) $\bar{x}\left(t_{0}\right)=x_{0}$.

To make our representation self-consistent we include some results on explicite ordinary differential equations.

\section{Global resulte for the Cauchy problem of ordinary differential equations}

Consider the system of ordinary differential equations

$$
d x / d t=f(x, t)
$$

under the following hypotheses:

(3.1) The function $f: \mathbf{R}^{n} \times J \rightarrow \mathbf{R}^{n}$ satisfies the Caratheodory condition.

(3.1) $)_{i}$ There are a constant $c$ and a function $m \in L^{1}\left(J, R^{+}\right)$such that

$$
|f(x, t)| \leq m(t)+c|x| \text { for all } x \in \mathbf{R}^{n} \text { and f.a.a. } t \in J \text {. }
$$

The hypotheses (3.1) $\mathrm{i}$ and (3.2) $)_{\mathrm{ii}}$ imply the following important property for the $\mathrm{Ne}$ myzki operator $F$ defined by $(F x)(t)=f(x(t), t)$.

Lemma 3.1[2]: Assume f satisfies the hypotheses $(3.1)_{\mathrm{i}}$ and $(3.2)_{\mathrm{ii}}$. Then $F$ is a continuous mapping from $L^{1}\left(J, R^{n}\right)$ into itself.

Further we suppose on $f$ the following hypothesis:

(3.1) $)_{\text {ii }}$ To any number $q, 0<q<1$, there is a norm $\|\cdot\|$ in $C\left(J, \mathbf{R}^{n}\right)$ with the properties

(a) $C\left(J, R^{n}\right)$ equipped with the norm $\|\cdot\|$ is a Banach space,

(b) the operator $\bar{F}: C\left(J, \mathbf{R}^{n}\right) \rightarrow C\left(J, \mathbf{R}^{n}\right)$ defined by

$$
\left(\bar{F}_{X}\right)(t)=\int_{t_{0}}^{t}\left(F_{X}\right)(s) d s=\int_{t_{0}}^{t} f(x(s), s) d s
$$

is strictly contractive with respect to the norm $\|\cdot\|$ with the contraction constant $q$.

The hypothesis $(3.1)_{\mathrm{iii}}$ can be fulfilled if $f$ satisfies for instance a condition of the type 


$$
\left|f\left(x_{1}, t\right)-f\left(x_{2}, t\right)\right| \leq \omega\left(\left|x_{3}-x_{2}\right|, t\right) \text { for all } x_{1}, x_{2} \in \mathbf{R}^{n} \text { and } t \in J,
$$

where $\omega: \mathbf{R}^{+} \times J \rightarrow \mathbf{R}^{+}$obeys the Caratheodory condition and the inequality

$$
\left\|\int_{t_{0}}^{t} \omega(\mid x(s) \|, s) d s\right\| \leq q\|. x\| \text { for all } x \in C\left(J, \mathbf{R}^{n}\right) \text { and } t \in J .
$$

In case $w(s, t)=s l(t)$ where $l \in L^{1}\left(J, \mathbf{R}^{+}\right)$we can choose the norm

$$
\|x\|=\max _{t \in J}\left\{\exp \left[-\alpha \int_{t_{0}}^{t} l(s) d s\right]|x(t)|\right\},
$$

where $\alpha$ is any positive number satisfying $\alpha>1$. Obviously, for $x \in C\left(J, \mathbf{R}^{n}\right)$ we have

$$
\begin{aligned}
& \left\|\int_{t_{0}}^{t} \omega(|x(s)|, s) d s\right\| \\
& \quad=\left\|\int_{t_{0}}^{t}|x(s)| l(s) d s\right\|=\max _{t \in J}\left\{\exp \left[-\alpha \int_{t_{0}}^{t} I(s) d s\right]\left|\int_{t_{0}}^{t} I(s)\right| x(s)|d s|\right\} \\
& \quad=\max _{t \in J}\left\{\exp \left[-\alpha \int_{t_{0}}^{t} l(s) d s\right]\left|\int_{t_{0}}^{t} I(s) \exp \left[\alpha \int_{t_{0}}^{s} I(\sigma) d \sigma\right] \exp \left[-\alpha \int_{t_{0}}^{s} I(\sigma) d \sigma\right]\right| x(s)|d s|\right\} \\
& \quad s\|x\| \max _{t \in J}\left\{\exp \left[-\alpha \int_{t_{0}}^{t} I(s) d s\right]\left|\int_{t_{0}}^{t} I(s) \exp \left[\alpha \int_{t_{0}}^{s} I(0) d \sigma\right] d s\right|\right\} \\
& \quad=\|x\| \max _{t \in J}\left\{\exp \left[-\alpha \int_{t_{0}}^{t} I(s) d s\right] \frac{1}{\alpha}\left[\exp \left[\alpha \int_{t_{0}}^{t} I(s) d s\right]-1\right]\right\} \leq \frac{1}{\alpha}\|x\| .
\end{aligned}
$$

that is, $q=1 / \alpha$ is arbitrary small provided $\alpha$ is sufficiently large.

Theorem 3.2: Assume the hypotheses (3.1) $)_{\mathrm{i}}-(3.1)_{\mathrm{iii}}$ hold. Then the Cauchy problem

$$
d x / d t=f(x, t), x\left(t_{0}\right)=x_{0}, t \in J
$$

has a unique $\mathrm{Ca}$-solution on $J$ where $T$ is any fixed positive number.

Proof: Let $X$ be the space $C\left(J, \mathbf{R}^{n}\right)$ equipped with the norm $\|\cdot\|$. We define the operator $F_{0}$ by

$$
\left(F_{0} x\right)(t)=x_{0}+(\bar{F} x)(t)=x_{0}+\int_{t_{0}}^{t} f(x(s), s) d s .
$$

By Lemma 3.1, the hypotheses $(3.1)_{\mathrm{i}}$ and $(3.1)_{\mathrm{ii}}$ imply $f(x(\cdot), \cdot) \in L^{1}\left(J, \mathbf{R}^{n}\right)$ for $x \in X$, thus we have $\bar{F} X \in A C\left(J, \mathbf{R}^{n}\right) \subset C\left(J, \mathbf{R}^{n}\right)$, that is $F_{\mathrm{o}} X \subset X$. Using the hypothesis $(3.1)_{\mathrm{iii}}$ we have

$$
\left\|F_{0}\left(x_{1}\right)-F_{0}\left(x_{2}\right)\right\| \leq q\left\|x_{1}-x_{2}\right\| \text { for all } x_{1}, x_{2} \in X,
$$

where $q$ can be assumed to be less than one. By Banach's fixed point theorem, $F_{0}$ has a unique fixed point $x^{\bullet}$ in $X$ which is a Ca-solution of (3.2)

Next we study the case where $f$ depends on some functional parameter $z$ and investigate the dependence of $x^{*}$ on $z$. The obtained result is used to establish an existence theorem for the Cauchy problem of some class of implicite differential equations.

Let us consider the initial value problem

$$
d x / d t=f_{1}(x, z(t), t), x\left(t_{0}\right)=x_{0}, t \in J
$$

assuming the following hypotheses:

(3.3)i The function $f_{1}: \mathbb{R}^{n} \times \mathbf{R}^{m} \times J \rightarrow \mathbf{R}^{n}$ satisfies the Caratheodory condition. 
(3.3) $\mathrm{ii}$ There are a constant $c_{1}>0$ and a function $m_{1} \in L^{1}\left(J, \mathbf{R}^{+}\right)$such that

$$
\left|f_{1}(x, z, t)\right| \leq m_{2}(t)+c_{1}(|x|+|z|) \text { for all }(x, z) \in \mathbb{R}^{\prime \prime} \times \mathbb{R}^{m} \text { and f.a.a. } t \in J \text {. }
$$

(3.3) $)_{i j}$ The function $z$ belongs to the space $L^{1}\left(J, R^{m}\right)$.

(3.3) iv There are positive numbers $q_{1}$ and $k_{1}$ where $q_{1}$ can be choosen arbitrarily small and a norm $\|\cdot\|$ in $C\left(J, R^{\prime \prime}\right)$ with the properties

(a) $C\left(J, R^{n}\right)$ equipped with the norm $\|\cdot\|$ is a Banach space,

(b) the operator $F_{1}: C\left(J, \mathbf{R}^{n}\right) \times L^{1}\left(J, \mathbf{R}^{m}\right) \rightarrow L^{2}\left(J, \mathbf{R}^{n}\right)$ defined by

$$
\left(F_{1}(x, z)\right)(t)=f_{1}(x(t), z(t), t)
$$

satisfies the relation

$$
\left\|\mid F_{1}(x, z)-F_{1}\left(x^{z}, z\right)\right\| \leq q_{1}\|x-\bar{x}\|+k_{1}\|z-\bar{z}\| \|
$$

for $x, \bar{x} \in C\left(J, \mathbf{R}^{n}\right)$ and $z, \bar{z} \in L^{1}\left(J, \mathbf{R}^{n}\right)$, where $\|1 \cdot 1\|$ is defined by

$$
\||z|\|=\left\|\int_{t_{0}}^{t}|z(s)| d s\right\| \text {. }
$$

In case that $f_{\mathrm{z}}$ fulfills the generalized Lipschitz condition

$$
\left|f_{1}\left(x_{1}, z_{1}, t\right)-f_{1}\left(x_{2}, z_{2}, t\right)\right| \leq \lambda_{1}(t)\left|x_{1}-x_{2}\right|+x_{1}(t)\left|z_{1}-z_{2}\right|
$$

for $x_{1}, x_{2} \in \mathbf{R}^{n}, z_{1}, z_{2} \in \mathbf{R}^{m}, t \in \mathbf{R}$, where $\lambda_{1} \in L^{1}\left(J, \mathbf{R}^{+}\right)$and $\left.x_{1} \in L^{\propto} q \mathbf{R}^{+}\right)$with ess sup $x_{1} \leq \bar{k}_{1}$, we can choose a norm in $C\left(J, \mathbf{R}^{n}\right)$ by

$$
\|x\|=\max _{t \in J}\left\{\exp \left[-\alpha \int_{t_{0}}^{t} \lambda_{1}(s) d s\right]|x(t)|\right\}, \alpha>1 .
$$

Then we have according to (3.6)

$$
\||z|\|=\max _{t c J}\left\{\exp \left[-\alpha \int_{r_{0}}^{t} \lambda_{1}(s) d s\right] \int_{r_{0}}^{t}|z(t)| d t\right\} .
$$

Thus, (3.5) holds with $q_{1} \leq 1 / \alpha$ and $k_{1} \leq \bar{k}_{1}$.

By Lemma 3.1, the hypotheses (3.3) $-(3.3)_{\mathrm{iv}}$ imply that $f(x, t):=f_{1}(x, z(t), t)$ satisfies the assumptons $(3.1)_{\mathrm{i}}-(3.1)_{\mathrm{iii}}$. Thus, to any $z \in L^{1}\left(J, R^{n}\right)$ the Cauchy problem (3.3) has a unique $\mathrm{Ca}$-solution denoted by $x_{z}$. Concerning the dependence of $x_{z}$ on $z$ we have the following result.

Theorem 3.3: Assume the hypotheses $(3.3)_{\mathrm{i}}-(3.3)_{\mathrm{iv}}$ are valid. Let $\mathrm{x}_{\mathrm{z}_{1}}, \mathrm{x}_{z_{2}}$ be the Ca-solutions of (3.3) to $z_{1}, z_{2} \in L^{1}\left(J, \mathbf{R}^{m}\right)$, respectively. Then we have

$$
\left\|x_{z_{1}}-x_{z_{2}}\right\| \leq k_{1} /\left(1-q_{1}\right)\left\|\left|z_{2}-z_{2}\right|\right\| \text {. }
$$

where $q_{1}$ can be supposed to be less than one.

Proof: The solutions $x_{z_{1}}$ and $x_{z_{2}}$ satisfy the integral equation

$$
y(t)=x_{0}+\int_{t_{0}}^{t} f(y(s), z(s), s) d s, t \in J .
$$

From (3.8) and (3.4) - (3.6) we get

$$
\left\|x_{z_{1}}-x_{z_{2}}\right\|=\left\|F_{1}\left(x_{z_{1}}, z_{1}\right)-F_{1}\left(x_{z_{2}}, z_{2}\right)\right\|\left\|\leq q_{1}\right\| x_{z_{1}}-x_{z_{2}}\left\|+k_{1}\right\|\left|z_{1}-z_{2}\right| \| .
$$

Since we may suppose $q_{1}<1$ we obtain from (3.9) the inequality (3.7) 
Now we apply this result to prove a global existence theorem for the Cauchy problem of a class of implicit differential equations

$$
d x / d t=f_{1}(x, d x / d t, t), x\left(t_{0}\right)=\lambda_{0}, t \in J .
$$

Theorem 3.4: Suppose $f_{1}$ satisfies the hypotheses $(3.3)_{\mathrm{i}},(3.3)_{\mathrm{ii}}$ and $(3.3)_{\mathrm{iv}}$. Under the additional assumption

$$
k_{2}<1
$$

the Cauchy problem (3.10) has a unique $C a-s o l u t i o n$ where $T$ is any fixed positive number.

Proof: Consider the Cauchy problem (3.3) in case $m=n$. By Theorem 3.2, to any $z \epsilon$ $L^{1}\left(J, \mathbf{R}^{n}\right)$ the problem $(3.3)$ has a unique solution $x_{z}$ on $J$. We define the operator $\widetilde{F}_{1}: L^{1}\left(J, \mathbf{R}^{n}\right)$ $\rightarrow L^{1}\left(J, \mathbf{R}^{\prime \prime}\right)$ by $\left(\widetilde{F}_{1} z\right)(t)=f_{1}\left(x_{z}(t), z(t), t\right)$. By $(3.4)-(3.6)$ we have for $z_{1}, z_{2} \in L^{1}\left(J, \mathbf{R}^{n}\right)$

$$
\left\|\left|\tilde{F}_{1}\left(z_{1}\right)-\tilde{F}_{1}\left(z_{2}\right)\left\|\leq q_{1}\right\| x_{z_{1}}-x_{z_{2}}\left\|+k_{1}\right\|\right| z_{1}-z_{2} \mid\right\| \leq k_{1} /\left(1-q_{1}\right)\left\|z_{1}-z_{2}\right\| \| .
$$

Hence, $\widetilde{F}_{1}$ is strictly contractive iff $k_{1}+q_{1}<1$. As we can choose $q_{1}$ arbitrarily small, the validity of (3.11) implies $\widetilde{F}_{1}$ to be strictly contractive on $L^{1}\left(J, \mathbf{R}^{m}\right)$. Therefore, $\widetilde{F}_{1}$ has a unique fixed point $z^{*}$ satisfying $d x_{z}-/ d t=f_{1}\left(x_{z}-(t), z^{*}(t), t\right)=z^{*}$, that means $(3.10)$ has a unique $\mathrm{Ca}-$ solution on $J$

\section{Global results for the Cauchy problem to a class of differential-algebraic equations}

Next we consider the Cauchy problem

$$
\begin{aligned}
& d x / d t=f_{1}(x, z(t), t) \\
& z(t)=g_{1}(x(t), z(t), t)
\end{aligned} \quad x\left(t_{0}\right)=x_{0}, t \in J
$$

under the following hypotheses:

(4.1) $)_{i}$ The functions $f_{1}: \mathbf{R}^{n} \times \mathbf{R}^{m} \times J \rightarrow \mathbf{R}^{n}, g_{1}: \mathbf{R}^{n} \times \mathbf{R}^{m} \times J \rightarrow \mathbf{R}^{m}$ obey the Caratheodory condition.

(4.1) $)_{\mathrm{ii}}$ There are a constant $\bar{c}_{1}>0$ and a function $\bar{m}_{1} \in L^{2}\left(J, \mathbf{R}^{+}\right)$such that $\left|f_{1}(x, z, t)+g_{1}(x, z, t)\right| \leq \bar{m}_{1}(t)+\bar{c}_{1}(|x|+|z|) \forall(x, z) \in \mathbf{R}^{n} \times \mathbf{R}^{m}$ and f.a.a. $t \in J$.

(4.1) ${ }_{\mathrm{iii}}$ The function $f_{1}$ satisfies the hypothesis $(3.3)_{\mathrm{iv}}$.

(4.1) $)_{\text {iv }}$ The function $g_{1}$ is such that the operator $G_{1}: C\left(J, \mathbf{R}^{n}\right) \times L^{1}\left(J, \mathbf{R}^{m}\right) \rightarrow L^{2}\left(J, \mathbf{R}^{m}\right) \mathrm{de}$ fined by $G_{1}(x, z)(t)=g_{1}(x(t), z(t), t)$ fulfills for all elements $x_{2}, x_{2} \in C\left(J, R^{n}\right)$ and $z_{1}, z_{2} \in L^{1}\left(J, R^{m}\right)$ the relation

$$
\|\left\{G_{1}\left(x_{1}, z_{1}\right)-G_{1}\left(x_{2}, z_{2}\right)\left\|\leq q_{2}\right\| x_{1}-x_{2}\left\|+k_{2}\right\|\left|z_{1}-z_{2}\right| \|\right. \text {. }
$$

Theorem 4.1: Assume the hypotheses $(4.1)_{\mathrm{i}}-(4.1)_{\mathrm{iv}}$ hold. If we additionally suppose $k_{2}<1$.

then the Cauchy problem (4.1) has a unique $C a$-solution on $J$ with any $T>0$.

Proof: By Theorem 3.3, the hypotheses $(4.1)_{i}-(4.1)_{i i i}$ imply that the differential equation in (4.1) has to any $z \in L^{1}\left(J, R^{m}\right)$ a unique Ca-solution $x_{z}$ satisfying $x_{z}\left(t_{0}\right)=x_{0}$. We define the operator $\widetilde{G}_{1}$ on $L^{1}\left(J, \mathbb{R}^{m}\right)$ by

$$
\left(\check{G}_{1} z\right)(t)=g_{1}\left(x_{z}(t), z(t), t\right)
$$


It is obvious that under our conditions $\widetilde{G}_{1}$ maps from $L^{1}\left(J, R^{m}\right)$ into $L^{1}\left(J, \mathbf{R}^{m}\right)$. From the relations (4.4) and (4.2) we get

$$
\left\|\left|\tilde{G}_{1} z_{1}-\tilde{G}_{1} z_{2}\left\|\leqslant \leq q_{2}\right\| x_{z_{1}}-x_{z_{2}}\left\|+k_{2}\right\|\right| z_{1}-z_{2}\right\| \| \text { for all } z_{1}, z_{2} \in L^{\prime}\left(J, R^{m}\right) .
$$

Taking into account (3.7) we obtain from (4.5)

$$
\left\|\left|\tilde{G}_{1} z_{1}-\tilde{G}_{1} z_{2}\right|\right\| s\left(k_{1} q_{2} /\left(1-q_{1}\right)+k_{2}\right)\left\|\left|z_{1}-z_{2}\right|\right\| \text {. }
$$

Thus, for sufficientely small $q_{2}$ the condition (4.3) implies that $\tilde{G}_{1}$ is strictly contractive on $L^{\prime}\left(J, R^{\prime \prime \prime}\right)$ and has a unique fixed point $z^{*}$. Therefore, the couple $\left(x_{z^{*}}, z^{*}\right)$ is a $\mathrm{Ca}-$ solution of (4.1) on $J$

In the next step we study the dependence of the solution $(\bar{x}, \bar{z})$ on some functional parameter. To this end we consider the problem

$$
\begin{aligned}
& d x / d t=F(x, z(t), y(t), t), \quad x\left(t_{0}\right)=x_{0}, t \in J \\
& z(t)=G(x(t), z(t), y(t), t)
\end{aligned}
$$

and assume the following hypotheses:

(4.6) $)_{\mathrm{i}}$ The function $y$ belongs to the space $L^{1}\left(J, R^{k}\right)$.

(4.6) ${ }_{\mathrm{ii}}$ The functions $F: \mathbf{R}^{n} \times \mathbf{R}^{m} \times \mathbf{R}^{k} \times J \rightarrow \mathbf{R}^{n}$ and $G: \mathbf{R}^{n} \times \mathbf{R}^{m} \times \mathbf{R}^{k} \times J \rightarrow \mathbf{R}^{m}$ satisfy the Caratheodory condition.

(4.6) ${ }_{\mathrm{iii}}$ There are a constant $\bar{c}>0$ and a function $\bar{m} \in L^{1}\left(J, \mathbf{R}^{+}\right)$such that

$$
|F(x, z, y, t)|+|G(x, z, y, t)| \leq \bar{m}(t)+\bar{c}_{1}(|x|+|z|+|y|)
$$

for all $(x, z, y) \in \mathbf{R}^{n} \times \mathbf{R}^{m} \times \mathbf{R}^{n}$ and f.a.a. $t \in J$.

(4.6) $)_{\mathrm{iv}}$ There are positive numbers $q_{i}, k_{i}, l_{i}(i=1,2)$ where $q_{i}$ can be choosen arbitrarily small and a norm $\|\cdot\|$ in $C\left(J, \mathbf{R}^{n}\right)$ such that the following conditions are satisfied:

(a) $C\left(J, \mathbf{R}^{n}\right)$ equipped with the norm $\|\cdot\|$ is a Banach space.

(b) The operator $F_{2}: C\left(J, \mathbf{R}^{n}\right) \times L^{1}\left(J, \mathbf{R}^{m}\right) \times L^{1}\left(J, \mathbf{R}^{k}\right) \rightarrow L^{1}\left(J, \mathbf{R}^{n}\right)$ defined by

$$
\left(F_{2}(x, z, y)\right)(t)=F(x(t), z(t), y(t), t)
$$

satisfies the relation

$$
\left\|\left|F_{2}(x, z, y)-F_{2}(\bar{x}, \bar{y}, \bar{z})\left\|\leq q_{1}\right\| x-\bar{x}\left\|+k_{1}\right\|\right| z-\bar{z}\left|\left\|+l_{1}\right\|\right| y-\bar{y} \mid\right\| .
$$

(c) The operator $G_{2}: C\left(J, R^{n}\right) \times L^{1}\left(J, \mathbf{R}^{m}\right) \times L^{1}\left(J, \mathbf{R}^{k}\right) \rightarrow L^{1}\left(J, \mathbf{R}^{m}\right)$ defined by

$$
\left(G_{2}(x, z, y)\right)(t)=G(x(t), z(t), t)
$$

satisfies the relation

$$
\left\|\left|G_{2}(x, z, y)-G_{2}(\bar{x}, \bar{y}, \bar{z})\left\|\leq q_{2}\right\| x-\bar{x}\left\|+k_{2}\right\|\right| z-\bar{z}\left|\left\|+l_{2}\right\|\right| y-\bar{y} \mid\right\| \text {. }
$$

(d) The relation (4.3) holds.

According to Theorem 4.1 the problem (4.6) has to any $y \in L^{1}\left(J, \mathbf{R}^{k}\right)$ a unique Ca-solution $\left(\bar{x}_{y}, \bar{z}_{y}\right)$. With respect to the dependence on $y$ we have the following result.

Theorem 4.2: Assume the hypotheses $(4.6)_{i}-(4.6)_{i}$ are valid. Then we have

$$
\begin{aligned}
& \left\|\bar{x}_{y_{1}}-\bar{x}_{y_{2}}\right\| \leq \gamma\left\|\mid y_{1}-y_{2}\right\| . \\
& \left\|\bar{z}_{y_{1}}-\bar{z}_{y_{2}}\left|\left\|\leq\left\{\frac{q_{2} \gamma+I_{2}}{1-k_{2}}\right\}\right\|\right| y_{1}-y_{2}\right\|, \text { where } \gamma=\frac{k_{1} l_{2}+l_{1}\left(1-k_{2}\right)}{\left(1-q_{1}\right)\left(1-k_{2}\right)-k_{1} g_{2}}
\end{aligned}
$$


Proof : Let $y_{1}, y_{2} \in L^{2}\left(J, R^{k}\right)$ be given. The corresponding solutions $\left(\bar{x}_{y_{1}}, \bar{z}_{y_{1}}\right) \cdot\left(\bar{x}_{y_{2}}, \bar{z}_{y_{2}}\right)$ of (4.6) satisfy the problem

$$
x(t)=x_{0}+\int_{t_{0}}^{t} f(x(s), z(s), y(s), s) d s, z(t)=g(x(t), z(t), y(t), t) .
$$

Using (4.7) and (4.8) we get from (4.13) the estimate

$$
\begin{aligned}
\left\|\bar{x}_{y_{1}}-\bar{x}_{y_{2}}\right\| & =\left\|F_{2}\left(\bar{x}_{y_{1}}, \bar{z}_{y_{1}}, y_{1}\right)-F_{2}\left(\bar{x}_{y_{2}}, \bar{z}_{y_{2}}, y_{2}\right)\right\| \| \\
& \leq q_{1}\left\|\bar{x}_{y_{1}}-\bar{x}_{y_{2}}\right\|+k_{1}\left\|\left|\bar{z}_{y_{1}}-\bar{z}_{y_{2}}\right|\right\|+l_{1}\left\|\mid y_{1}-y_{2}\right\| .
\end{aligned}
$$

For $q_{1}<1$ we obtain from $(4.14)$ the estimate

$$
\left\|\bar{x}_{y_{1}}-\bar{x}_{y_{2}}\right\| \leq k_{1} /\left(\begin{array}{ll}
1 & q_{1}
\end{array}\right)\left\|\bar{z}_{y_{1}}-\bar{z}_{y_{2}}\left|\left\|+4 /\left(1-q_{1}\right)\right\|\right| y_{1}-y_{2}\right\| .
$$

Taking into account (4.9), (4.10) and (4.3) we get from (4.13) the estimate

$$
\left\|\left|\bar{z}_{y_{1}}-\bar{z}_{y_{2}}\left\|\leq a_{2} /\left(1-k_{2}\right)\right\| \bar{x}_{y_{2}}-\bar{x}_{y_{2}}\left\|+z_{2} /\left(1_{1}-k_{2}\right)\right\|\right| y_{1}-y_{2}\right\| .
$$

Substituting (4.16) into (4.15) we obtain the estimate

$$
\left\|\bar{x}_{y_{1}}-\bar{x}_{y_{2}}\right\| \leq \frac{k_{1}}{1-q_{1}}\left\{\frac{q_{2}}{1-k_{2}}\left\|\bar{x}_{y_{1}}-\bar{x}_{y_{2}}\right\|+\frac{l_{2}}{1-k_{2}}\left\|\mid y_{1}-y_{2}\right\|\right\}+\frac{l_{1}}{1-q_{1}}\left\|\mid y_{1}-y_{2}\right\| .
$$

For sufficiently small $q_{2}$ the inequality (4.11) follows immediately from (4.17). Using (4.11) we get from (4.16) the inequality (4.12)

Finally we consider the Cauchy problem for the class of differential-algebraic equations

$$
\begin{aligned}
& d x / d t=F(x, z(t), d x / d t, t), \quad x\left(t_{0}\right)=x_{0}, t \in J . \\
& z(t)=G(x(t), z(t), d x / d t, t)
\end{aligned}
$$

Using Theorem 4.2 we can prove the existence and uniqueness of a solution to (4.18).

Theorem 4.3: Assume the hypotheses $(4.6)_{\mathrm{ii}}-(4.6)_{\mathrm{iv}}$ with $n=k$ are valid. Additionally we assume

$$
k_{2}<1, l_{1}+k_{1} l_{2} /\left(1-k_{2}\right)<1 .
$$

Then the Cauchy problem (4.18) has a unique Ca-solution on $J$, where $T$ is any positive number.

Proof: To $y \in L^{1}\left(J, R^{n}\right)$ given, by Theorem 4.1 the problem (4.6) has a unique solution $\left(\bar{x}_{y}, \bar{z}_{y}\right)$. We define the operator $F_{3}$ on $L^{1}\left(J, R^{n}\right)$ by

$$
\left(F_{3} y\right)(t)=F\left(\bar{x}_{y}(t), \bar{z}_{y}(t), y(t), t\right) \text {. }
$$

Under the hypotheses above we have $F_{3}: L^{1}\left(J, \mathbf{R}^{n}\right) \rightarrow L^{1}\left(J, \mathbf{R}^{n}\right)$. We shall prove that $F_{3}$ is also strictly contractive under the same conditions. Let $y_{1}, y_{2} \in L^{1}\left(J, \mathbf{R}^{n}\right)$. By (4.7), (4.8) we get from (4.20)

$$
\left\|F_{3}\left(y_{1}\right)-F_{3}\left(y_{2}\right)\right\| \leq q_{1}\left\|\bar{x}_{y_{1}}-\Gamma_{y_{2}}\right\|+k_{1}\left\|\left|\bar{z}_{y_{1}}-\bar{z}_{y_{2}}\right|\right\|+I_{1}\left\|\left|y_{1}-y_{2}\right|\right\| \text {. }
$$

By using (4.11) and (4.12) it follows

$$
\left\|F_{3}\left(y_{2}\right)-F_{3}\left(y_{2}\right)\right\| \leq\left\{q_{1} \gamma+\frac{k_{1} q_{2} \gamma}{1-k_{2}}+\frac{k_{1} i_{2}}{1-k_{2}}+I_{1}\right\}\left\|\left|y_{1}-y_{2}\right|\right\| \text {. }
$$

Since $q_{1}$ and $q_{2}$ may be choosen arbitrarily small and since $\gamma$ is uniformly bounded for 
decreasing $q_{1}$ and $q_{2}$ the condition (4.19) implies that $F_{3}$ is strictly contractive on $L^{1}\left(J \cdot R^{\prime \prime}\right)$ and has a unique fixed point $y^{\bullet}$ in $L^{1}\left(J, R^{n}\right)$. Then the couple $\left(\bar{x}_{y_{*}}, \bar{z}_{y_{\bullet}}\right)$ is the unique $\mathrm{Ca}$ solution of $(4.18)$

\section{Numerical approximation}

As mentioned in the introduction, an important tool for the numerical integration of a broad class of differential-algebraic equations (1.1) in case $n+m$ to be large is the wave form relaxation method. This approach represents an iteration method in some function space, the essential feature of which is to decompose the large system (1.1) into a set of subsystems each of which is integrated independently on $J$ and taking into account inputs from other subsystems from their state on the previous iteration [3,5]. This process is equivalent to rewrite the problem (1.1) as

$$
\begin{aligned}
& d \xi / d t=\tilde{f}(\xi, \xi, \zeta, d \xi / d t, t) . \quad \xi\left(t_{0}\right)=x_{0}, t \in J \\
& \zeta=\tilde{g}(\xi, \xi, \zeta, d \xi / d t, t)
\end{aligned}
$$

where $\xi \in \mathbf{R}^{n}, \zeta \in \mathbf{R}^{m}$, and to choose an iteration scheme providing a sequence of approximations converging to the solution of (5.1). Theorem 4.3 can be easily extended to the Cauchy problem (5.1).

Theorem 5.1: Assume the following hypotheses:

(5.1) The functions $\tilde{f}: \mathbf{R}^{n} \times \mathbf{R}^{n} \times \mathbf{R}^{m} \times \mathbf{R}^{n} \times J \rightarrow \mathbf{R}^{n}$ and $\tilde{g}: \mathbf{R}^{n} \times \mathbf{R}^{n} \times \mathbf{R}^{m} \times \mathbf{R}^{n} \times J \rightarrow$ $\mathbf{R}^{m}$ obey the Carathéodory condition.

(5.1) 11 There are a constant $\tilde{c}$ and a function $\tilde{m} \in L^{4}\left(J, R^{*}\right)$ such that

$$
|\widetilde{f}(w, x, z, y, t)|+|\widetilde{g}(w, x, z, y, t)| \leq \tilde{m}(t)+\tilde{c}(|w|+|x|+|z|+|y|)
$$

for all $(w, x, z, y) \in \mathbf{R}^{\prime \prime} \times \mathbf{R}^{\prime \prime} \times \mathbf{R}^{m} \times \mathbf{R}^{\prime \prime}$ and f.a.a. $t \in J$.

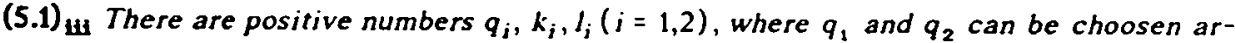
bitrarily small, and a norm $\|\cdot\|$ in $C\left(J, \mathbf{R}^{n}\right)$ such that the following conditions are satisfied:

(a) $C\left(J, \mathbf{R}^{n}\right)$ equipped with the norm $\|\cdot\|$ is a Banach space.

(b) The operator $\widetilde{F}_{2}: C\left(J, \mathbf{R}^{n}\right) \times C\left(J, \mathbf{R}^{n}\right) \times L^{4}\left(J, \mathbf{R}^{m}\right) \times L^{2}\left(J, \mathbf{R}^{n}\right) \rightarrow L^{4}\left(J, \mathbf{R}^{n}\right)$ defined by

$$
\left(\tilde{F}_{2}(w, x, z, y, t)\right)(t)=\tilde{f}(w(t), x(t), z(t), y(t), t)
$$

satisfies the relation

$$
\begin{aligned}
& \left\|\mid \hat{F}_{2}(w, x, z, y)-\tilde{F}_{2}(\bar{w}, \bar{x}, \bar{z}, \bar{y})\right\| \\
& \quad \leq q_{1}\left(\left\|w-\overline{w^{\prime}}\right\|+\|x-\bar{x}\|\right)+k_{1}\||z-\bar{z}|\|+l_{1}\|! y-\bar{y} \mid\| .
\end{aligned}
$$

(c) The operator $\widetilde{G_{2}}: C\left(J, \mathbf{R}^{n}\right) \times C\left(J, \mathbf{R}^{n}\right) \times L^{2}\left(J, \mathbf{R}^{m}\right) \times L^{1}\left(J, \mathbf{R}^{n}\right) \rightarrow L^{4}\left(J, \mathbf{R}^{n}\right)$ defined by

$$
\left(\widetilde{G}_{2}(w, x, z, y, t)\right)(t)=\widetilde{g}(w(t), x(t), z(t), y(t), t)
$$

satisfies the relation

$$
\begin{aligned}
& \left\|\widetilde{G}_{2}(w, x, z, y)-\tilde{G}_{2}(\bar{w}, \bar{x}, \bar{z}, \bar{y})\right\| \\
& \quad \leq q_{2}(\|w-\bar{w}\|+\|x-\bar{x}\|)+k_{2}\|z-\bar{z}\|+I_{2}\||y-\bar{y}|\| .
\end{aligned}
$$


(5.1) iv The relations (4.19) hold.

Then the Cauchy problem (5.1) has a unique Ca-solution on $J$ where $T$ is any positive number.

The Proof of this theorem proceeds in the same way as for Theorem 4.3

In what follows we prove that the hypotheses of Theorem 5.1 guaranteeing the existence of a unique solution $\left(\xi^{*}, \zeta^{*}\right)$ of $(5.1)$ also ensures that this solution can be iteratively approximated by a wave-form relaxation algorithm. To this end we consider the iteration scheme

$$
\begin{aligned}
\xi^{k}(t) & =\xi_{0}+\int_{t_{0}}^{t} \tilde{f}\left(\xi^{k}(s), \xi^{k-1}(s), \zeta^{k} 1(s), \eta^{k}{ }^{1}(s), s\right) d s, \\
\zeta^{k}(t) & =\widetilde{g}\left(\zeta^{k}(s), \xi^{k-1}(s), \zeta^{k-1}(s), \eta^{k-1}(s), s\right), \\
\eta^{k}(t) & =\tilde{f}\left(\xi^{k}(s), \xi^{k-1}(s), \zeta^{k-1}(s), \eta^{k-1}(s), s\right) .
\end{aligned}
$$

Let us set $S=C\left(J, \mathbf{R}^{n}\right) \times L^{1}\left(J, \mathbf{R}^{m}\right) \times L^{2}\left(J, \mathbf{R}^{n}\right)$. Under the assumptions of Theorem 5.1 , the scheme (5.6) defines an operator $T$ mapping the space $S$ into itself. We introduce a norm $\|l \cdot\|_{a, b}$ in $S$ by

$$
\left\|\left|(\xi, \zeta, \eta)\|\|_{a, b}=a\|\xi\|+b\||\zeta|\|+\||\eta|\|\right.\right. \text {. }
$$

where $a$ and $b$ are any positive numbers. The space $S$ equipped with that norm is a Banach space. In the sequel we establish the existence of numbers $a$ and $b$ such that $T$ is strictly contractive with respect to the norm $\|1 \cdot \mid\|_{a, b}$.

Theorem 5.2: Assume the hypotheses of Theorem 5.1 are satisfied. Then there are positive numbers $a$ and $b$ such that the sequence $\left\{\left(\xi^{k}, \zeta^{k}, \eta^{k}\right)\right\}$ defined by $(5.6)$ converges with respect to the norm $\|1 \cdot\| \|_{a, b}$ to the unique solution $\left(\xi^{*}, \zeta^{*}\right)$ of $(5.1)$ for any initial guess in $S$.

Proof: Using the abbreviation $\Delta^{k+1} v=v^{k+1}-v^{k}$ we get from (5.6), (5.2), (5.3) and (4.2)

$$
\begin{aligned}
\left\|\Delta^{k+1} \xi\right\| & =\left\|\tilde{F}_{2}\left(\xi^{k+1}, \xi^{k}, \zeta^{k}, \eta^{k}\right)-\tilde{F}_{2}\left(\xi^{k}, \xi^{k-1}, \zeta^{k-1}, \eta^{k-1}\right)\right\| \| \\
& \leq q_{1}\left(\left\|\Delta^{k+1} \xi\right\|+\left\|\Delta^{k} \xi\right\|\right)+k_{1}\left\|\left|\Delta^{k} \zeta\right|\right\|+l_{1}\left\|\mid \Delta^{k} \eta\right\| .
\end{aligned}
$$

For $q_{1}<1$ it follows from (5.8)

$$
\left\|\left|\Delta^{k+1} \xi\right|\right\| \leq \frac{q_{1}}{1-q_{1}}\left\|\Delta^{k} \xi\right\|+\frac{k_{1}}{1-q_{1}}\left\|\left|\Delta^{k} \zeta\right|\right\|+\frac{l_{1}}{1-q_{2}}\left\|\mid \Delta^{k} \eta\right\| .
$$

By a similar way we obtain

$$
\left\|\left|\Delta^{k+1} \eta\right|\right\| \leq \frac{q_{1}}{1-q_{1}}\left\|\Delta^{k} \xi\right\|+\frac{k_{1}}{1-q_{1}}\left\|\left|\Delta^{k} \zeta\right|\right\|+\frac{l_{1}}{1-q_{1}}\left\|\left|\Delta^{k} \eta\right|\right\| .
$$

From (5.6), (5.4), (5.5) and (5.9) it follows

$$
\left\|\Delta^{k+1} \zeta\left|\left\|\leq \frac{q_{2}}{1-q_{1}}\right\| \Delta^{k} \xi\left\|+\frac{k_{2}\left(1-q_{2}\right)+q_{2} k_{1}}{1-q_{1}}\right\|\right| \Delta^{k} \zeta\left|\left\|+I_{2} \frac{k_{2}\left(1-q_{1}\right)+q_{2} k_{1}}{1-q_{1}}\right\|\right| \Delta^{k} \eta\right\| .
$$

Let us set $\omega=(\xi, \zeta, \eta)$ and $a=2\left(b q_{1}+q_{2}\right)$ in (5.7). Then we get from (5.7) - (5.9) 


$$
\begin{aligned}
\left(1-q_{1}\right)\left\|\left|\Delta^{k+1} \omega\right|\right\|_{a, b}= & \left(2\left(b q_{1}+q_{2}\right) q_{1}+b q_{2}+q_{1}\right)\left\|\Delta^{k} \xi\right\| \\
& +\left(2\left(b q_{1}+q_{2}\right) k_{1}+b\left(k_{2}+q_{2} k_{1}-k_{2} q_{1}\right)+k_{1}\right)\left\|\left|\Delta^{k} \zeta\right|\right\| \\
& +\left(2\left(b q_{1}+q_{2}\right) l_{1}+b\left(l_{2}+q_{2} l_{1}-l_{2} q_{1}\right)+l_{1}\right)\left\|\mid \Delta^{k} \eta\right\| .
\end{aligned}
$$

From (5.10) it follows that if there exists a positive number $b$ satisfying the inequalities

$$
b k_{2}+k_{1}<b \text { and } b I_{2}+l_{1}<1
$$

then there are positive numbers $q_{0}$ and $x \in(0,1)$ such that for $q_{1}, q_{2}<q_{0}$ the relations

$$
\begin{aligned}
& \left(2\left(b q_{1}+q_{2}\right) k_{1}+b\left(k_{2}\left(1-q_{1}\right)+q_{2} k_{1}\right)+k_{1}\right) \leq\left(1-q_{1}\right) \times b \\
& \left(2\left(b q_{1}+q_{2}\right) k_{1}+b\left(l_{2}\left(1-q_{1}\right)+q_{2} l_{1}\right)+l_{1}\right) \leq\left(1-q_{1}\right) x
\end{aligned}
$$

hold. The inequalities (5.11) are equivalent to $k_{1} /\left(1-k_{2}\right)<b<\left(1-l_{1}\right) / l_{2}$. It is easy to show that the relations (4.19) holding accordingly to the hypothesis $(5.1)_{\text {iv }}$ imply $k_{1} /\left(1-k_{2}\right)$ $<\left(1-l_{1}\right) / l_{2}$. Thus, there is a positive number $b$ satisfying $(5.12)$. To such a number $b$ there is a number $q^{\bullet}<q_{0}$ such that, for $q_{1}, q_{2}<q^{*}$, the inequality

$$
\left(2\left(b q_{1}+q_{2}\right) q_{1}+b q_{2}+q_{1}\right) \leq\left(1-q_{1}\right) \times 2\left(b q_{1}+q_{2}\right)
$$

holds. Therefore, we have

$$
\left\|\left|\Delta^{k+1} \omega\right|\right\|_{A, b} \leq x\left\|\left|\Delta^{k} \omega\right|\right\|_{a, b} \text { for } q_{1}, q_{2}<q^{*} \text {. }
$$

Hence, the sequence $\left\{\left(\xi^{k}, \zeta^{k}, \eta^{k}\right)\right\}$ converges with respect to the norm $\|1 \cdot 1\|_{a, b}$ to an element $\left(\xi^{*}, \zeta^{*}, \eta^{*}\right) \in S$ for any initial guess in $S$ where $\left(\xi^{*}, \zeta^{*}\right)$ is the unique solution of (5.1)

Remark 5.3: Theorem 5.2 generalizes similar results obtained in $[3,5]$.

\section{REFERENCES}

L1] BORCHARDT; J., SCHNEIDER; K.R., and M. UHLE : Elektriksimulation mit Relaxationsverfahren. In: 32. Internat. Wiss. Kolloq., Techn. Hochschule Ilmenau (Hrsg.: W. Kemnitzer). Heft 1 (1987), $11-14$.

[2] Fučlk, S.: Solvability of Nonlinear Equations and Boundary Value Problems. Dortrecht: Reidel Publ. 1980.

[3] Lelarasmee, E., RUEHLI, A.E., and A.L. SANGiovaNni-VinCentelli: The wave-form rolaxation for time-domain analysis of large-scale integrated circuits. IEEE Trans. CAD-1 (1982), 131 - 145.

[4] NEWTON, A.R., and A.L. SANGIOVANNI - VINCENTELLI : Relaxation based electrical simulation. IEEE Trans. ED - 30 (1983). 1184 - 1207.

[5] SCHNEIDER, K.R.: A note on the waveform relaxation method. Systems Analysis, Modelling, Simulation (to appear).

[6] SPIRO, H.: Simulation integrierter Schaltungen durch universelle Rechenprogramme. Muinchen: Oldenbourg 1985.

Received 09.11.1989; in revised form 23.05.1990

Author's address:

Dr. K.R. Schneider

Karl-Weierstrass - Institut fur Mathematik

Mohrenstr. 39

D (Ost) 1086 - Berlin 